\title{
Towards high speed and low power silicon photonic data links.
}

\author{
David J. Thomson ${ }^{1}$, Kapil Debnath ${ }^{1,4}$, Weiwei Zhang ${ }^{1}, \mathrm{Ke} \mathrm{Li}^{1}$, Shenghao Liu ${ }^{1}$, Fanfan Meng ${ }^{1}$, Ali Z Khokhar ${ }^{1}$, \\ Callum Littlejohns ${ }^{1}$, James Byers ${ }^{2}$, Lorenzo Mastronardi ${ }^{1}$, Muhammad K. Husain ${ }^{2}$, Frederic Y. Gardes ${ }^{1}$, Shinichi \\ Saito ${ }^{2}$, Xia Chen ${ }^{1}$, Milan M. Milosevic ${ }^{1}$, Yohann Franz ${ }^{1}$, Antoine F. J. Runge ${ }^{1}$, Sakellaris Mailis ${ }^{1}$, Anna C. Peacock ${ }^{1}$, \\ Peter Wilson ${ }^{3}$ and Graham T. Reed ${ }^{1}$
}

1 - Optoelectronics Research Centre, University of Southampton, Southampton, UK

2 - Faculty of Physical Sciences and Engineering, University of Southampton, Southampton, UK

3 - Department of Electronic and Electrical Engineering, University of Bath, UK

4 - Department of Electronics \& Electrical Communication Engineering, Indian Institute of Technology, India

Abstract: Silicon is a low-cost material platform in which it is possible to form photonic integrated circuits serving a number of applications from optical communications to sensing. For optical communications applications, e.g. short reach data communication within data centres, the optical modulator is a key component with its capabilities having a significant bearing on the overall performance of the photonic circuit. In this paper we report our recent work on optical modulators in silicon and their integration with CMOS electronics on silicon.

Introduction: Global demand for bandwidth is relentlessly growing fuelled by more and more data hungry applications. To support this demand a large number of high bandwidth photonic links are required. Silicon photonics is one leading technology in this application area. Silicon as material basis with which to form the photonic integrated circuits (PICS) required for data communication is advantageous from a cost perspective since the fabrication can be done in a CMOS manner (high volume and yield). Another advantage of silicon are the prospects for the close integration of electronics and photonics. In order to send data in the optical domain using a silicon PIC, an optical modulator is required. At the University of Southampton we are carrying out research into a wide range of photonic technologies to tackle challenges in this application and others. In this paper, we present a snapshot of our recent research activity in the area of silicon optical modulators.

Silicon optical modulators: There have been numerous demonstrations on high speed optical modulator in silicon in recent years. Different mechanisms have been employed to overcome one of silicon's shortcomings as a photonic material, the lack of a strong electro-optic effect. Approaches involving the use of other photonic materials with the silicon waveguide for example III-V's [1], SiGe [2], polymer [3] and LiNbO3 [4], have demonstrated good performance, however in this case CMOS compatibility and/or fabrication simplicity are compromised to an extent. The more popular approach has generally been to make used of the free carrier plasma dispersion effect in silicon. This effect describes the change in refractive index and absorption that occurs with changes in electron and hole densities in the material as formularised by Nedeljkovic et al. [5]. Electrically controlled modulation has been achieved using this effect then by employing electronic structures in the waveguide in order to change the free carrier concentrations where the light propagates. The three main approaches that have been followed are carrier injection in a forward biased PIN diode, carrier depletion in a reverse biased PN diode and a carrier accumulation around a capacitor like structure [6]. Carrier injection devices are efficient and require simple fabrication but the modulation rate is limited to a few $\mathrm{Gbit} / \mathrm{s}$ if pre-emphasis driving is not used. Carrier depletion structures provide high data rate modulation and require again relatively simple fabrication but the efficiency is low meaning that a power hungry travelling wave drive approach is generally required together with a large footprint. The carrier accumulation approach combines a high efficiency and high speed operation, however the fabrication 
is more complicated particularly the challenge of forming device with single crystal silicon on either side of the dielectric layer. The use of polysilicon has been demonstrated [7-10] however polysilicon has inferior optical and electrical properties. The other challenge is to produce the structure in a 'standard' rib structure to allow ease of interfacing with other components or incorporating the phase shifter within another photonic structure for example a ring resonator of slow wave waveguide. At Southampton an approach has been developed to enable the formation of a vertical dielectric layer in the centre of a rib waveguide with single crystal silicon on either side [11]. Here we present results from a first fabrication run of carrier accumulation silicon optical modulator formed with this process [12]. The phase modulator is incorporated with modulation up to 20G bit/s as shown in figure 1.

(a)

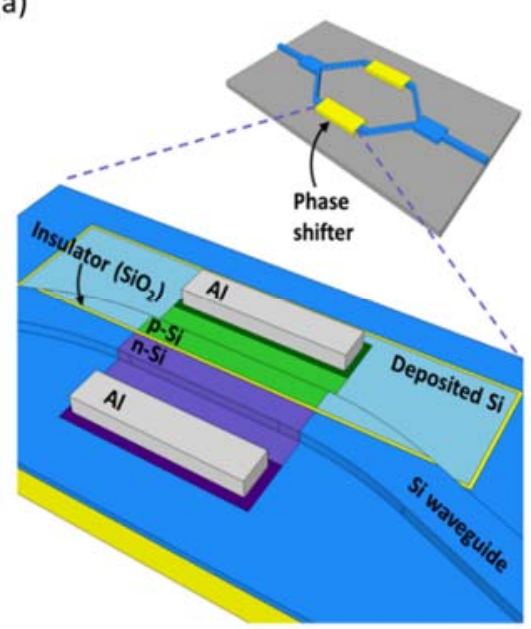

(b)

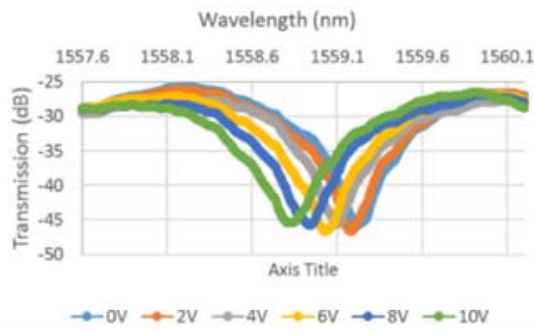

(c)

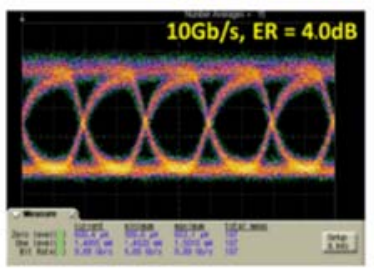

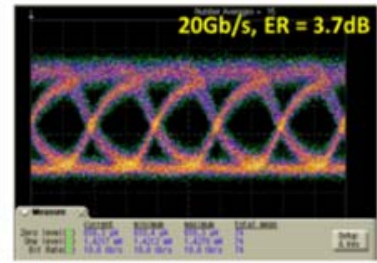

Figure 1 - Diagram of MOSCAP device structure (a), DC modulation (b) and high speed modulation (c) [12].

Elimination of phase tuning power: The scale of silicon waveguides used these days is generally on the order of a few hundred nanometres in both width and height. This together with the high index contrast means that devices become strongly susceptible to fabrication variations. Mach-Zehnder modulator (MZM) are generally preferred over ring based modulators because a symmetrical MZM is insensitive to global temperature changes and also less sensitive to fabrication variations, however the phase error induced by small variation in the waveguide dimensions between arms of the MachZehnder structure means that practically a tuning element is still required to ensure that the MZM is operated at the quadrature point. The addition of the tuning element increases the complexity and operating power for the MZM and is therefore undesirable. At Southampton we have developed a method to enable trimming of photonic device post fabrication to compensate for any fabrication errors and fix a device performance as required [13].

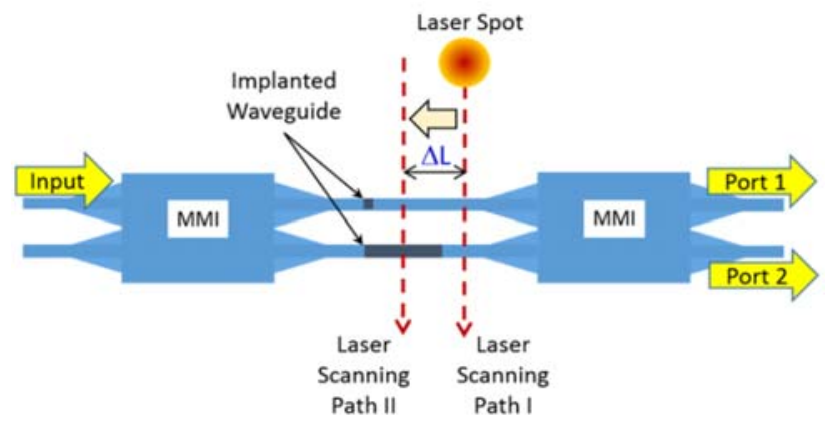

Figure 2 -Diagram of trimming process used for Mach-Zehnder modulators [13]. 
In our approach as depicted in figure 2, we first amorphise a section of the waveguide with the implantation of $\mathrm{Ge}$ ions, which causes an increase in the refractive index and then use localised laser annealing to recover the refractive index to close to that of single crystal silicon. To demonstrate our approach test Mach-Zehnder (MZ) structures have been fabricated and amorphous sections formed in both arms as depicted in figure 2. The optical transmission from both ports of the $\mathrm{MZ}$ with light coupled into the input port was then measured before and after different lengths of the amorphous waveguide in one arm of the MZ were exposed to laser annealing. Some example results are shown in figure 3 where it can be seen that in excess of a $\pi$ phase shift can be created between two MZ arms with a $5 \mu \mathrm{m}$ length of amorphous waveguide annealed demonstrating the validity of our approach. Clearly this technology can also be applied to other silicon photonic devices to fix performance errors resulting from fabrication variations.

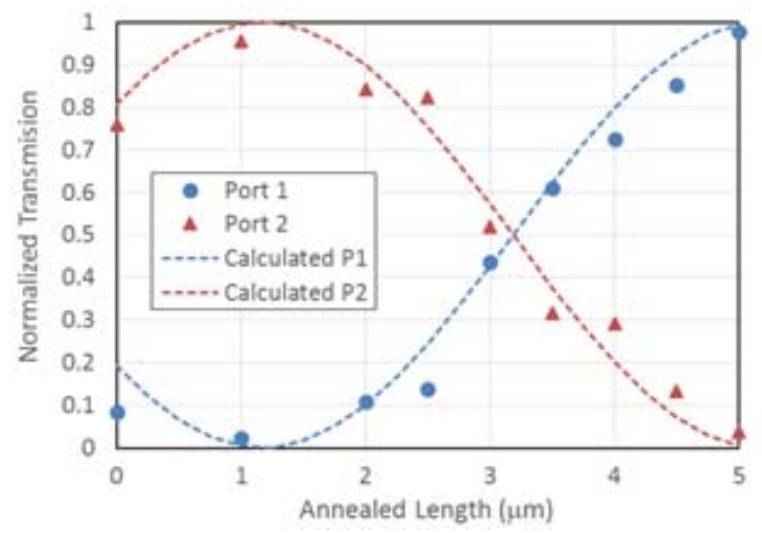

Figure 3 - Results of MZI transmission versus amorphous silicon waveguide annealing length [13].

Integration of high speed silicon optical modulators with CMOS drivers: An important consideration with regards the performance of the optical modulator is the integration of an electrical drive amplifier. The amplifier is required to provide sufficient drive voltage to the optical modulator in order to obtain the required modulation depth. The design and properties of each element, together with the integration technique used requires consideration in order to obtain a high performance. Different approaches have been demonstrated to integrate the driver including frontend integration which involves combining the two technologies on the same chip as part of the fabrication process. Such an approach can provide optimal performance without degradation due to parasitics from the bond pads and/or wires. Assembly effort is also saved in not having to perform integration post fabrication. However, forming large photonic components in high cost electronic real estate is not preferable from an economic perspective. Furthermore using separate chips for the photonics and electronics gives flexibility over the technology nodes which can be used in each case. In Southampton we have concentrated on this separate chip approach, with the electronic chips designed in house but fabricated in MPW services. Photonic chips have then been generally designed and fabricated in house, with either flip-chip or wire bonding to perform the electrical integration. Here we present some of our recent work in this area. An example is shown in figure 4 where we are using a wire bonding approach to integrate a carrier depletion based silicon optical modulator with a CMOS drive amplifier formed in the $65 \mathrm{~nm}$ TSMC CMOS node. Optical modulation up to $30 \mathrm{Gbit} / \mathrm{s}$ was shown in this case [14].

Summary: Silicon optical modulators are essential components within the next generation of low cost photonic integrated circuits to serve short reach data communication applications. In order to achieve a high performance in terms of modulation bandwidth, power efficiency etc... the design of the 
modulator, electronic drive amplifier, integration approach and phase tuning technique need to be considered carefully. In this work we have demonstrated a new approach to form a MOSCAP modulator featuring a vertical dielectric layer in the centre of the optical waveguide with single crystal silicon on either side.

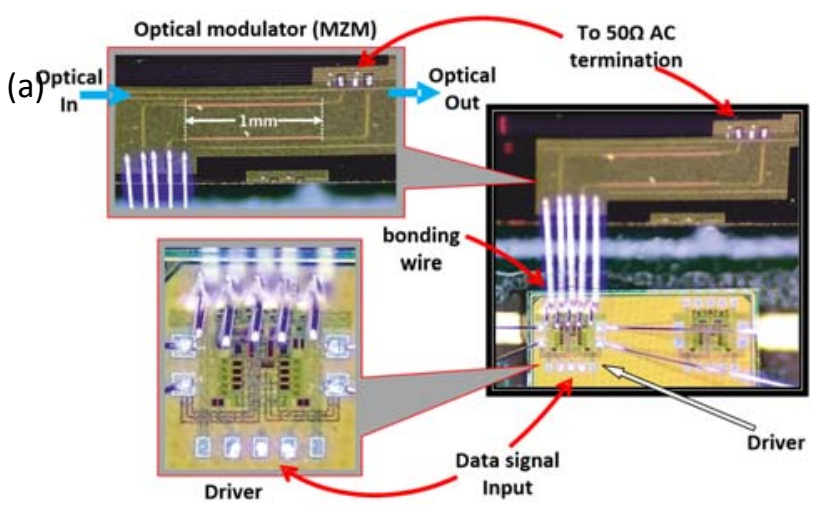

(b)

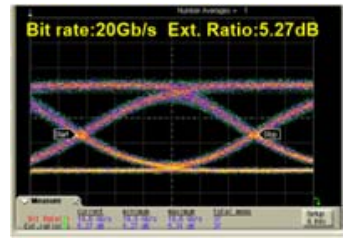

Figure 4-Microscope images of the optical modulator and driver integrated by wire bonding (a) and optical data showing modulation up to 30Gbit/s (b) [14].

Such an approach can lead to high efficiency and high speed modulation with low optical losses. We have also demonstrated a trimming technique which can accurately correct for fabrication errors in a MZI structure. This technique can eliminate the requirement to include a thermal phase tuning element which contributes to the complexity and power consumption of the modulator. Finally, we have presented our work on CMOS drivers and their integration with optical modulators.

Acknowledgements: G. T. Reed is a Royal Society Wolfson Merit Award holder and is grateful to both the Royal Society and the Wolfson Foundation for funding the award. D. J. Thomson acknowledges funding from the Royal Society for his University Research Fellowship. This work is supported by EPSRC Standard Grant (EP/M009416/1), EPSRC Manufacturing Fellowship (EP/M008975/1), EPSRC Platform Grant (EP/N013247/1), EPSRC Prosperity Partnership (EP/R003076/1), EPSRC Programme Grant (EP/L00044X/1), EU FP7 Marie-Curie Carrier-Integration-Grant (PCIG13-GA-2013-618116), University of Southampton Zepler Institute Research Collaboration Stimulus Fund, and Hitachi.

\section{References:}

[1] Y. B. Tang et al. Optics Express, vol. 20, no. 10, pp. 11529-11535, May 7, 2012.

[2] L. Mastronardi et al. Optics Express, vol. 26, Issue 6, pp. 6663-6673 (2018).

[3] C. Koos et al. 2014 European Conference on Optical Communication, Mo.3.4.5, 2014.

[4] L. Chen et al. Optica, vol. 1, no. 2, pp. 112-118, Aug 20, 2014.

[5] M. Nedeljkovic et al. IEEE Photonics Journal, vol. 3, no. 6, pp. 1171-1180, Dec, 2011.

[6] G. T. Reed et al. Nature Photonics, vol. 4, pp. 518-526, 2010.

[7] A. Liu et al. Nature 427, 615-618, 2004.

[8] M. Webster et al. IEEE Group IV Photonics, vol. 1, pp. 1-2, 2014.

[9] A. Shastri et al. IEEE Journal of Lightwave Technology, vol. 33, pp. 1255-1260, 2015.

[10] M. Sodagar et al. Optics Express vol. 23, pp. 28306, 2015.

[11] K.Debnath, et al. IEEE Photonics Technology Letters 29.15 (2017): 1269-1272.

[12] K. Debnath et al. Photonics Research vol. 6, no. 5, pp. 373-379, 2018.

[13] X. Chen et al. Photonics Research, vol. 5, pp. 578-582, 2017

[14] K. Li et al. IEEE Radio Frequency Integrated Circuits Symposium (RFIC 2015), 17-19 May, 2015. 